\title{
The genetic variation and covariation among fitness components in Drosophila melanogaster females and males
}

\author{
N. Tucić, \\ D. Cvetković and \\ D. Milanović
}

Institute of Zoology, Faculty of Science, University of
Belgrade, Studentski trg 16, 11000 Belgrade,
Yugoslavia.

The results presented in this study indicate low to moderate level of heritable variation for the following fitness components in Drosophila melanogaster sampled from natural population: early and late fecundity of females, virility of males and longevity of females and males. The most striking exception from this are high additive genetic variances for preadult developmental duration in both sexes. Females exhibit significant negative genetic correlations between early and late components of fitness. In contrast to females, we did not observe any measurable correlations between components of fitness in males. Our data suggest that genetic covariance structure between fitness components differs by sex. We also observed significant negative correlations between virility of males and late components of female life history.

\section{INTRODUCTION}

In 1957 Williams suggested that genes with beneficial effects during the first stages and pleiotropic deleterious effects in later periods of life history would be favoured by natural selection. According to Williams's theory, there should be a negative genetic correlation between early and late components of fitness. The assumption of negative genetic correlation among life history fitness components is also pivotal to the theory of life history evolution (see, e.g., Charlesworth, 1980).

Until very recently the supportive data for the evolutionary theory of life history were almost absent. Several recent studies (e.g., Rose and Charlesworth, 1981; Hegmann and Dingle, 1982; Luckinbill and Clare, 1985; Roach, 1986) have provided evidence of negative genetic correlation among traits at early and late stages of life history. However, Giesel et al. (1982), Stearns (1983), Murphy et al. (1983), Bell (1984), and MitchellOlds (1986) found no evidence of negative correlations among fitness components. Several studies that fail to show negative genetic correlations are methodologically flawed (see e.g., Reznick, 1985). In addition to these controversial results, the extent of fitness heritability in real populations is uncertain (see, e.g., Partridge, 1983 and Mitchell-Olds and Rutledge, 1986). Obviously, more experi- mental work on natural populations is needed to assess the true relationships among fitness components.

This study was designed to measure the extent of fitness heritability, as well as the structure of genetic correlations among fitness components in Drosophila melanogaster females and males sampled from a natural population. The following fitness components were measured: early and late female fecundity, virility of males, preadult developmental duration and longevity of females and males.

\section{MATERIAL AND METHODS}

A sample of about 200 females and 200 males of Drosophila melanogaster was collected in August 1986 at Valjevo (about $80 \mathrm{~km}$ south of Belgrade, Yugoslavia), and allowed to produce progeny under laboratory conditions. Virgin females were sampled from this base population. In order to preclude the possibility of developmental-rate bias in the flies collected, virgin flies were collected over 3 days. Then, 22 males ("sires") and 110 females ("dams") were chosen at random and mated, one male to five females. These 22 groups of five females and a single male of the same age (up to 5 days old).were kept in food vials for two days to allow mating. After mating each female 
was provided with a new laying vial every 4 hours during a period of 12 hours (i.e., three lots of eggs were collected in separate vials from each mated female). After removing the flies, the eggs were counted. Larvae were raised without density control. The duration of development from deposition of eggs to emergence of adults was measured. Adults were scored every 4 hours.

Female fecundity was measured in two periods during the life span of the flies: 2-10 days and 20-26 days after eclosion. During each egg-laying period the procedure was as follows. Eight newly emerged adult females, chosen at random from each dam, were provided with eight males and kept as pairs for one day to allow mating. After mating, each pair was placed in a food vial (of about $40 \mathrm{ml}$ ) and allowed to lay eggs during the following 48 hours. This procedure was repeated four times by placing flies into new vials for the next 48 hours. The second period of egg-counting started with the same females aged 20 days. This laying period covered 6 days. Female fecundity within each laying period is defined as the number of eggs laid per female per day.

Virility of males was measured as follows. The individual 11-day-old experimental male (which previously spent 10 days with his full sister from the fecundity experiment) and one $e$ (ebony body colour; the recessive marker on the third chromosome) male of the same age and virgin status were kept in a mating chamber (about $60 \mathrm{ml}$ food bottle) with four 5-day-old virgin $e$ females. All six flies were placed in a mating chamber without etherisation. After 10 hours in the mating chambers, the parent flies were recounted and females placed individually in food vials (experimental males were returned into the original vials). The progenies in each vial were later scored to indicate the type of male with which the female had mated: wild type progeny implied an experimental male, whereas $e$ progeny implied an $e$ male. This experimental procedure was done with 4 experimental males per each dam. The virility (V) of experimental males relative to the control males was estimated using the method of Haldane (1956; see also Brittnacker, 1981). The nested analysis of variance was done on the data following the $\arcsin \sqrt{V /(V+1)}$ transformation.

For longevity measurements, experimental females and males were kept together as pairs. New males or females were added to each vial with asynchronous death of the mates. The medium was changed weekly. The number of flies which died was counted every day. Longevity is defined as the life span of adults, from eclosion until death.
All cultures were maintained in the incubator at $25^{\circ} \mathrm{C}$ and 60 per cent humidity, using a yeast medium for Drosophila (Ohba, 1961). All handling was performed at room temperature, using ether anaesthesia.

The phenotypic variance components were estimated using the conventional formulae for sib analyses (Becker, 1967; Falconer, 1981). Since our data are unbalanced (unequal number of offspring per dam and of dams per sire), exact significance tests for the estimated genetical parameters cannot be made. Even though the exact significance tests are not available, the test for the unequal sizes in a nested design suggested by Falconer (1963) was used in the present paper.

\section{RESULTS}

Table 1 gives the main results of the sib analyses for different fitness components in $D$. melanogaster females and males. The effects of epistasis and linkage have been assumed to be negligible for these analyses. The patterns for the most traits suggest that between-dam variances are greater than the between-sire components, indicating a substantial bias due to non-additive genetic variance, or variance due to common environment. Therefore, as suggested by Falconer (1981), only the sire component can be used to estimate heritability of fitness components.

The variance could be partitioned into additive genetic variance $\left(V_{A}\right)$, dominance variance $\left(V_{D}\right)$ and two forms of environmental variance; $V_{E c}$, due to common environment between full sib families and $V_{E w}$, environmental variance within full sib families (Falconer, 1981). The estimates of the variance components in table 1 are based on the assumption of significant $V_{D}$ and negligible $V_{D}$, and that $V_{E w}=0$. Although we estimated the maximum possible value for $V_{D}$, it is interesting to compare $V_{D}$ and $V_{A}$ for different fitness components. The most striking finding from these comparisons are the relatively small $V_{D}$ values for preadult developmental duration for both sexes. The largest $V_{D} / V_{A}$ ratios were found for longevity of females and males. This is reflected, of course, in the estimations of heritability of these fitness components. The present experiment suggests that heritability for fitness components in $D$. melanogaster varies from 0.03 (for female longevity) to 0.91 (for preadult developmental time of the females). Heritabilities of all fitness components except preadult developmental duration are not significantly different from zero. Most 
Table 1 Estimated variance components for different fitness traits in $D$. melanogaster females and males based on the assumption of significant or negligible dominance genetic variance. $V_{P}=$ phenotypic variance, $V_{A}=$ additive variance, $V_{D}=$ dominance variance, $V_{E c}=$ common environmental variance, $V_{E w}=$ within full sib family variance. Narrow sense heritability $\left(h^{2}\right)$ included, standard error in parentheses

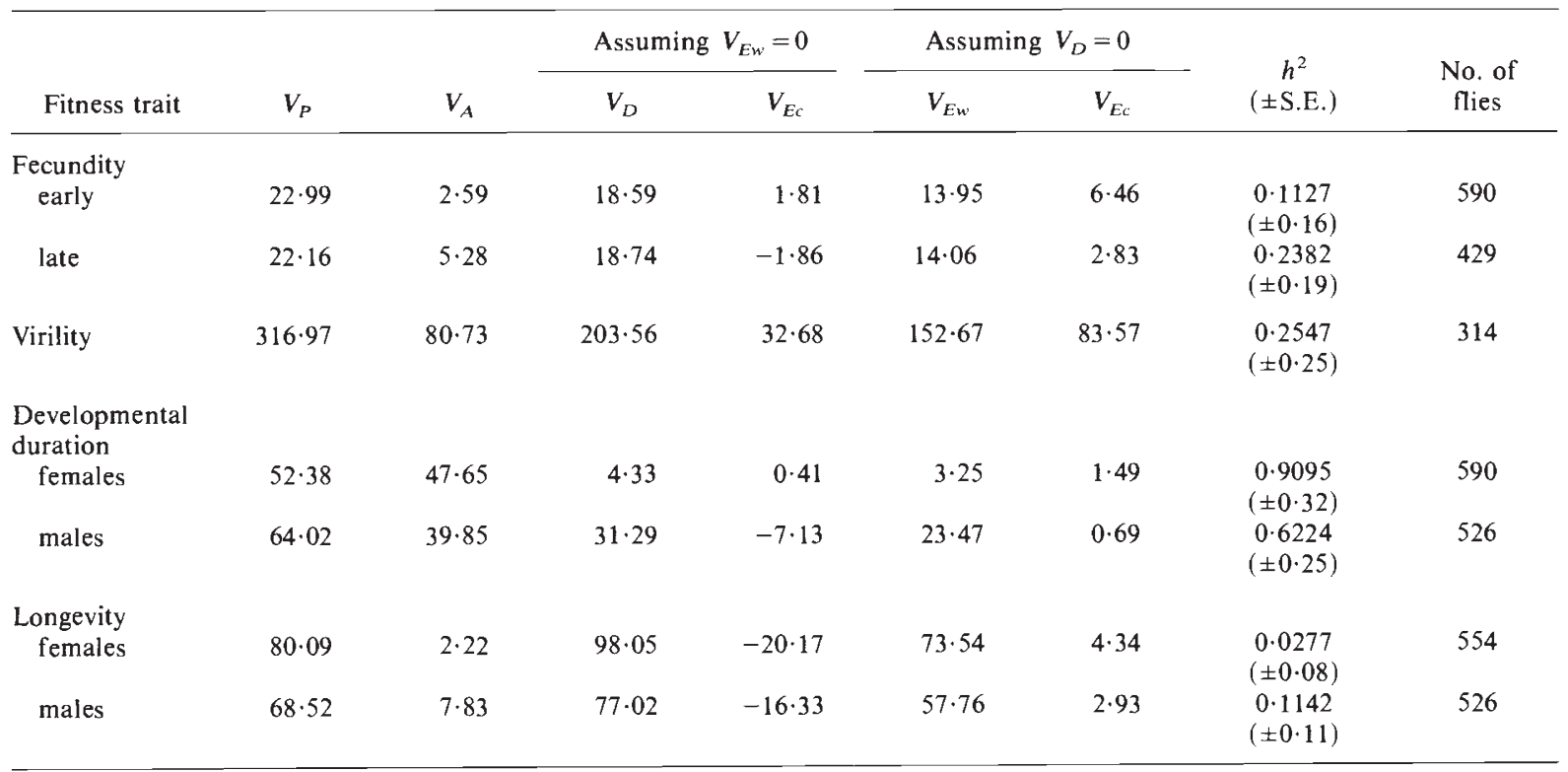

heritability estimates were in the low to moderate range $(<0 \cdot 3)$. It is also worth emphasising that heritability of late fecundity is about twice that of the heritability of fecundity at early ages (i.e., $0 \cdot 24$ vs. $0 \cdot 11$ ), mainly due to increasing additive genetic variance.

Phenotypic and genetic correlations among female and male fitness components are shown in table 2. The general impressions to be gained are negative correlations among early and late components of fitness in D. melanogaster females; i.e., early fecundity is negatively correlated with late fecundity $\left(r_{G}=-0.96, P<0.01\right)$ as well as with longevity $\left(r_{G}=-1.76, P<0.01\right)$. In other words, females which reproduce intensively at early age do so at the expense of longevity. Genetic and phenotypic correlations suggest that longevity is significantly associated with late fecundity. These findings are consistent with the observation of Rose and Charlesworth (1981) who found a similar relationship between longevity and early and late fecundity in $D$. melanogaster females.

Our data also suggest a positive genetic correlation between preadult developmental duration and longevity of females $\left(r_{G}=0.87, P<0.01\right)$. The genetic correlation between the length of preadult development and late fecundity has a negative statistically significant value $\left(r_{G}=-1.08, P<\right.$ $0 \cdot 01)$. While it appears that early fecundity has a nonantagonistic relationship with developmental rate, the absence of statistical significance of correlation coefficients (both phenotypic and genetic) suggests that this result must be taken with a caution. Table 2 also gives the estimates of phenotypic and genetic correlations between different fitness components of $D$. melanogaster males. In contrast to females, in males no genetic correlations were significant.

Table 2 Estimated genetic $\left(r_{G}\right)$ and phenotypic $\left(r_{P}\right)$ correlations in $D$. melanogaster females $(F)$ and males $(M)$

\begin{tabular}{|c|c|c|}
\hline Traits & $r_{G}$ & $r_{P}$ \\
\hline $\begin{array}{l}\text { Developmental duration and } \\
\text { Early fecundity (F) } \\
\text { Late fecundity (F) } \\
\text { Longevity (F) } \\
\text { (M) }\end{array}$ & $\begin{aligned} 0 \cdot 2149 \\
-1 \cdot 0789^{* *} \\
0 \cdot 8705^{* *} \\
-0 \cdot 3889\end{aligned}$ & $\begin{array}{l}-0.1207 \\
-0.1598 \\
1.4149^{* *} \\
0.0382\end{array}$ \\
\hline $\begin{array}{l}\text { Early fecundity and } \\
\text { Late fecundity (F) } \\
\text { Longevity (F) }\end{array}$ & $\begin{array}{l}-0.9633^{* *} \\
-1.7637^{* *}\end{array}$ & $\begin{array}{l}-0.7657^{* *} \\
-0.8772^{* *}\end{array}$ \\
\hline $\begin{array}{l}\text { Late fecundity and } \\
\text { Longevity (F) }\end{array}$ & $0.8610^{* *}$ & 0.2624 \\
\hline $\begin{array}{l}\text { Virility and } \\
\text { Developmental duration (M) } \\
\text { (F) }\end{array}$ & $\begin{array}{r}0.3314 \\
-0.4839\end{array}$ & 0.0308 \\
\hline $\begin{array}{l}\text { Longevity (M) } \\
\qquad(\mathrm{F}) \\
\text { Early fecundity (F) } \\
\text { Late fecundity (F) }\end{array}$ & $\begin{array}{c}0 \cdot 1603 \\
-1 \cdot 4595^{* *} \\
0.2655 \\
-1.0086^{* *}\end{array}$ & 0.1233 \\
\hline
\end{tabular}

$* * P<0.01$ 
An interesting question for evolutionary ecologist is the influence of male mating success on the female fitness components. Our results (table 2) suggest that there are negative correlations between virility of males and female late fecundity $\left(r_{G}=-1 \cdot 01, P<0 \cdot 01\right)$ or longevity $\left(r_{G}=-1 \cdot 46\right.$, $P<0 \cdot 01)$. We found no significant genetic correlation between virility and female preadult developmental duration, as well as early fecundity. It is important to note, that genetic correlations between virility of males and female fitness components were calculated by regressing the average values of female fitness components on the virility data characterising each dam within sires. Since the correlation of full sibs means include a within full sibs error component, this is a biased estimate of genetic correlation.

\section{DISCUSSION}

The magnitude and signs of genetic parameters in natural populations are still unresolved issues of evolutionary biology. The expectation that populations at selective equilibria have little or no heritable variation for fitness has come down from Fisher's Fundamental Theorem (Fisher, 1930). However, interpretation of heritability data as well as genetic correlations among characters with large effects on fitness is not straightforward. For instance, Fisher's observation of decreasing additive genetic variance concerns total fitness, but not fitness components. If so, we should expect, as pointed out by Falconer (1981), "additive genetic correlations between characters that are major components of fitness to be negative" (p. 306). This conclusion arises from elementary consideration: gene alleles which give rise to positive genetic correlations among fitness components should be driven toward fixation. Thus, it could be expected that in a large panmictic population at selective equilibrium any remaining genetic covariance among major fitness components will be mostly negative. In such populations, therefore, negative genetic correlations among fitness components could be an important mechanism for the maintenance of genetic variation. On the other hand, as predicted by quantitative genetics theory, any local populational subdivision with some level of inbreeding will cause positive genetic correlations among major fitness components. This sort of genetic correlation may be generated by inbreeding if deleterious recessive alleles have negative pleiotropic effects in all fitness components.
The results presented here indicate low-tomoderate level of heritable variation for the most analysed fitness components in D. melanogaster females and males. The presented data are also consistent with the hypothesis that the largest component of genetic variation for fitness is dominance variance (see, e.g., MacKay, 1986). The most striking exceptions from this are high additive genetic variances for egg-to-adult developmental time in both sexes (table 1). The high level of additive variance for preadult developmental duration presumably reflects the effects of stabilising selection for a fluctuating and, temporally uncertain, intermediate optimum (see Istock, 1983, for further discussion). The alternative explanation, that this level of additive variance for developmental time is due to disruptive selection, although not impossible, seems less likely. Prout (1962) showed that in D. melanogaster the increase in developmental time variance with disruptive selection did not involve an increase in additive genetic variance. Our estimations of heritability for longevity, female fecundity and preadult developmental duration are in accordance with those of Tantawy and El-Helw (1970). They also showed little or no heritable variation for fecundity and longevity, whereas the additive variance of preadult developmental time was very high.

Our fecundity data seem to be contradictory to those obtained by Rose and Charlesworth (1981). Their findings suggest that the narrow sense heritability of fecundity falls from $0 \cdot 3-0 \cdot 4$ at early ages to $0 \cdot 2-0 \cdot 3$ at later ages. Our data showed the opposite trend; at early age our estimate was about $0 \cdot 11$ but at later age it increased to $0 \cdot 23$. In addition, we did not observe any change in environmental variance across different age classes of fecundity. Although small sample size necessitate caution in interpretation, these results are interesting. It seems conceivable that genes affecting late fecundity have smaller effects in wild Drosophila populations than those acting at earlier ages, but in special circumstances (as in the case of the overwintering adults) it is very likely that fecundity at later ages might be significant. It is intriguing, therefore, to consider the possibility that agedependent effects on heritability may have been selected to favour continued reproduction by older parents, since their progeny might be better adapted to changes in environmental conditions due to greater genetic variability. Our findings that heritability of fecundity increases with advancing age of females are parallel to those for heritabilities of meristic characters in Drosophila and guppy fish 
Poecelia obtained by Beardmore et al. (1975) and Beardmore and Shami (1976).

The male reproductive components of fitness have been measured in a female-choice, male mating competition experiment. Thus, "virility" (Prout, 1971a) is a composite aspect of fitness, involving physiological and behavioural abilities of males, as well as their actual performance under competitive conditions. The estimated heritability for virility is about twice the size of heritability for the fecundity at early ages (i.e., $0 \cdot 25$ vs. $0 \cdot 11$ ). This indicates that genetic variation due to virility could be greater than that due to female reproductive component at about same age of the flies. It is consistent with results of Prout $(1971 a, b)$, Bungaard and Christiansen (1972) and Anderson et al. (1979), who showed that both in laboratory and in wild populations of Drosophila a large amount of selection could occur among males. Brittnacker (1981) showed that chromosome homozygosity in D. melanogaster and D. pseudoobscura had a more pronounced effect on virility than on viability or female reproductive component.

The studies of life history evolution ignore males altogether. The underlying assumption is that aspects of the male life history are not correlated with female fitness components. However, empirical evidence for this assumption are almost absent. Brittnacker (1981) found no significant correlation between virility and female weight (which is an index of female fecundity). This author also found no correlation between viability and virility of males when he compared different chromosomal homozygotes.

We did not observe any measurable correlation between virility and developmental time or longevity of males. Interestingly enough, females exhibit significant negative genetic correlations between early and late components of fitness. It seems, therefore, that genetic covariance structure between fitness components differs by sex.

At present, our data do not enable us to offer any conceivable hypothesis on the mechanism involved in the expression of sex differences in covariance structure between fitness components. In general, however, the covariance structure may differ between sexes because some autosomal genes are expressed differentially in the hormonal environment of each sex (Robertson, 1959). Alternatively, the specific genotype by sex interaction arises when some genes influencing expression of fitness components occur on the $\mathrm{X}$ chromosome. Since the chromosome in $D$. melanogaster contains about 20 per cent of the haploid genome and because the level of dosage compensation in this Genus could complicate expression of polygenes (see e.g., Cowley et al., 1986), clarifying the underlaying factors is important to understanding the sex differences in covariance structure between fitness components.

Although there is a clear need for replications of these types of studies, significant negative correlations between virility of males and late components of female life history (i.e., late fecundity and longevity) lead to the tentative conclusion that variation in male mating success could be, in part, responsible for the evolution of the antagonistic relationship between early and late components of fitness observed in female individuals.

Acknowledgements The authors are grateful to an anonymous reviewer for suggesting improvements to an earlier draft of the paper.

\section{REFERENCES}

ANDERSon, W. W., LeVine, L., OLIVERA, O., POWELL, J. R., DE LA ROSA, M. E., SALCEDA, V. M., GASO, M. I., AND GUZ MAN, J. 1979. Evidence for selection by male mating success in natural populations of Drosophila pseudoobscura. Proc. Nat. Acad. Sci. U.S.A., 76, 1519-1523.

BEARDMORE, J. A., LINTS, F. A. AND AL-BALDAWI, A. I. F. 1975. Parental age and heritability of sternopleural chaeta number in Drosophila melanogaster. Heredity, 34, 71-82.

BEARDMORE, J. A. AND SHAMI, S. A. 1976. Parental age, genetic variation and selection. Karlin, S. and Nevo, E. (eds.) In Population Genetics and Ecology, Academic Press, New York.

BECKER, W. A. 1967. Manual of Procedures in Quantitative Genetics, Wash. State Univ. Press, Washington.

BELL, G. 1984. Measuring the cost of reproduction. II. The correlation structure of the life tables of five freshwater invertebrates. Evolution, 38, 314-326.

BRITTNACKER, J. G. 1981. Genetic variation and genetic load due to the male reproductive component of fitness in Drosophila. Genetics, 97, 719-730.

BUNDGAARD, J. AND CHRISTIANSEN, F. B. 1972. Dynamics of polymorphisms. I. Selection components in an experimental population of Drosophila melanogaster. Genetics, $71,439-460$.

CHARLEWSWORTH, B. 1980. Evolution in Age-structured Populations, Cambridge University Press, Cambridge.

COWley, D. E., ATCHLEY, W. R. AND RUTLEDGE, J. J. 1986 Quantitative genetics of Drosophila melanogaster. I. Sexual dimorphism in genetic parameters for wing traits. Genetics, $114,549-566$.

FALCONER, D. S. 1963. Quantitative inheritance. In Burdette, W. J. (ed.) Methodology in Mammalian Genetics, HoldenDay, Inc. San Francisco.

FALCONER, D. S. 1981. Introduction to Quantitative Genetics, Longman, London and New York.

FISHER, R. A. 1930. The Genetical Theory of Natural Selection, Clarendon Press, Oxford. 
HALDANE, J. B. S. 1956. The estimation of viabilities. J. Genetics, 54, 294-296.

HEGMANN, J. P. AND DINGLE, H. 1982. Phenotypic and genetic covariance structure in milkweed bug life history. In Dingle, H. and Hegmann, J. P., (eds.) Evolution and Genetics of Life Histories, Springer-Verlag, New York.

ISTOCK, C. A. 1983. The extent and consequences of heritable variation for fitness characters. In King, C. E. and Dawson, P. S. (eds.) Population Biology, Retrospect and Prospect, Columbia University Press, New York.

LUCKINBILL, L. S. AND CLARE, M. J. 1985. Selection for life span in Drosophila melanogaster. Heredity, 55, 9-18.

MACKAY, T. F. C. 1986. A quantitative genetic analysis of fitness and its component in Drosophila melanogaster. Genet. Res., $47,59-70$.

MITCHELL-OLDS, T. AND RUTLEDGE, J. J. 1986. Quantitative genetics in natural plant populations: A review of the theory. Amer. Nat., 127, 379-402.

MURPHY, P. A., GIESEL, J. T. AND MANLOVE, M. N. 1983. Temperature effects on life history variation in Drosophila simulans. Evolution, 37, 1181-1191.

OHBA, S. 1961. Analytical studies on the experimental populations of Drosophila. I. The effect of larvae populational density upon the pre-adult growth in Drosophila melanogaster and Drosophila virilis. Biol. J. Okayama University, 7,87 .

PARTRIDGE, L. 1983. Non-random mating and offspring fitness. In Bateson, P. (ed.) Mate Choice, Cambridge University Press, Cambridge.
PROUT, T. 1962. The effects of stabilizing selection on time of development in Drosophila melanogaster. Genet. Res., 3, 364-382.

PROUT, T. 1971 $a$. The relation between fitness components and population prediction in Drosophila. I. The estimation of fitness components. Genetics, 68, 127-149.

PROUT, T. $1971 \mathrm{~b}$. The relation between fitness components and population prediction in Drosophila. II. Population predic. tion. Genetics, 68, 151-167.

REZNICK, D. 1985. Costs of reproduction: an evaluation of the empirical evidence. Oikos, 44, 257-267.

ROACH, D. A. 1986. Life history variation in Geranium carolinianum. 1. Covariation between characters at different stages of the life cycle. Amer. Nat., 128, 47-57.

ROBERTSON, A. 1959. The sampling variance of the genetic correlation coefficient. Biometrics, 15, 469-485.

ROSE, M. R. AND CHARLESWORTH, B. 1981. Genetics of life history in Drosophila melanogaster. I. Sib analysis of aduit females. Genetics, 97, 173-186.

STEARNS, S. C. 1983. The genetic basis of differences in life history traits among six populations of mosquitofish ( $\mathrm{Gam}$ busia affinis) that shared ancestors in 1905. Evolution, 37, 618-627.

TANTAWY, A. O. AND EL-HELW, M. R. 1970. Studies on natural populations of Drosophila. IX. Some fitness components and their heritabilities in natural and mutant populations of Drosophila melanogaster. Genetics, 64, 79-81.

WILliaMS, G. C. 1957. Pleiotropy, natural selection and the evolution of senescence. Evolution, 11, 398-411. 